\title{
The Continuous Recombination of Codification and Personalisation KM strategies: A Retrospective Study
}

\author{
Ettore Bolisani ${ }^{1}$, Antonella Padova ${ }^{2}$ and Enrico Scarso ${ }^{1}$ \\ ${ }^{1}$ DTG, University of Padova, Italy \\ ${ }^{2} \mathrm{KM}$ Consultant, Milan, Italy \\ ettore.bolisani@unipd.it \\ enrico.scarso@unipd.it \\ DOI: 10.34190/EJKM.18.02.008
}

\begin{abstract}
It is increasingly considered important to understand how companies plan their Knowledge Management (KM) strategy. The literature provides evidence that there may be different possible approaches to KM strategy. A significant distinction has been made between "codification" and "personalization". Sometimes, these two approaches have been seen to be alternative to one another. In other cases scholars argued that a company can follow a strategy that mixes the two approaches depending on diverse intertwined factors. Still, on this topic, the literature provides various and sometimes contrasting results that need clarification and confirmation. Especially, there is the need to understand if changes in internal and external conditions may induce modifications in a firm's KM strategy. The goal of the study is to analyse how the mix of codification and personalisation can vary over time in the same company, due to changing organizational and environmental conditions. With this purpose, the evolution of KM initiatives of a multinational company was investigated. The findings of the study confirm that the strategic mix can change over the years due to modifications in the factors of the company's internal and external context. Furthermore, the case shows that the different factors have different weight and play a different role in influencing such changes. Specifically, in the investigated case, the factors related to the competitive context affected the evolution of the KM strategy more significantly than internal factors (which were just enablers or constraints of the evolutionary path). In addition, the study shows that this classic distinction between codification and personalization may not be easy to use in practical terms, due to the complexity of KM activities and needs in a company: this point can represent a fresh start of a future research agenda.
\end{abstract}

Keywords: KM strategy, Codification, Personalisation, Case study, Longitudinal study

\section{Introduction}

The notion of Knowledge Management (KM) strategy, intended as the long-term planning of organizational, managerial and technical resources that a company employs for its KM programmes (Bolisani and Bratianu, 2018) has increasingly attracted the attention of academics and practitioners (Holsapple and Jones, 2006, Coakes et al., 2010; Bolisani and Bratianu, 2017). Its definition is still questioned and not unanimously accepted (Shannak et al., 2012), but the basic idea is that there are different possible approaches for a company to managing knowledge and to planning KM activities. A significant number of studies attempted to categorize possible KM strategy approaches: one of the most popular classification makes a distinction between - codification and personalization (Hansen et al., 1999; Greiner et al., 2007; Ajith Kumar and Ganesh, 2011) - that are generally intended to be opposite to one another. However, many scholars affirm that they are complementary: a company can follow a mix of the two approaches, whose "optimal ratio" may vary (Venkitachalam and Willmott, 2017). This point deserves additional research, particularly to clarify what organizational or strategic factors can induce a company to adopt a particular combination of codification and personalization. In addition, the extant empirical analyses generally consider case studies (of one or more companies) at a specific point in time: conversely, it would be interesting to explore if and how the KM strategic mix of the same company changes over time.

This is the goal of the present study: to analyse how the combination of codification and personalisation can vary over time in the same company. By examining this, it becomes possible to clarify the factors that lead the company to adopt a particular KM approach, and how the changing organizational, strategic, and environmental conditions induce modifications in that approach. With this purpose, the evolution of KM programs of a multinational company was investigated.

Reference this paper: Bolisani, E., Padova, A., and Scarso, E., 2020. The Continuous Recombination of Codification and Personalisation KM strategies: A Retrospective Study. The Electronic Journal of Knowledge Management, 18(2), pp. 185195, available online at www.ejkm.com 
The findings of the study contribute to improve our understanding of the factors that may induce a particular KM strategy mix. In addition, they also prompt fresh reflections on the practical usefulness of the distinction between codification and personalization.

The paper is organized as follows. The next section introduces the conceptual background. Then, the research questions are formulated, and the research method is illustrated. Section four presents the case study, and the main findings are thoroughly discussed in section five. The last section summarizes the theoretical and practical implications, and the limitations of the study.

\section{Theoretical background}

From the very beginning, two contrasting perspectives on knowledge and KM have coexisted: the objectivistic and the practice-based views (Hislop et al., 2018), that strictly derive from the popular distinction between tacit and explicit knowledge (Polanyi, 1966; Nonaka and Takeuchi, 1995). It can be argued that these perspectives influenced the whole KM discipline, and especially the studies of KM strategies: indeed, the distinction between codification and personalization KM strategy (Hansen et al., 1999; Greiner et al., 2007; Ajith Kumar and Ganesh, 2011) stems from the two mentioned perspectives. The codification strategy focuses on a people-to-document approach: it is assumed that the main goal of KM is to capture, codify, store, disseminate and reuse explicit knowledge in a form that is useful for achieving a company's organizational goals. This strategy requires major investments in information technology (IT), since complex IT systems are needed to store and disseminate the "objects" (i.e. documents, data, etc.) that contain the codified knowledge. The economic model that underlies codification is the reuse economics: investing once in a knowledge asset and then reusing it many times (Hansen et al., 1999). The personalization (or human-oriented KM strategy) focuses on a person-to-person approach and has the goal to promote the sharing of tacit knowledge through networking and interactions, essentially between people. IT plays a minor role, and its goal is, primarily, to facilitate conversations between individuals, and their exchange of (tacit) knowledge. The economic model that underlies personalisation is the expert economics (Hansen et al., 1999) that emphasizes the role of the human element. Powell and Ambrosini (2012), on the basis of a multiple-case study, summarize the main features that distinguish the personalization and codification approaches (Table 1).

Table 1: Comparison of KM approaches (adapted from Powell and Ambrosini, 2012)

\begin{tabular}{|l|l|l|}
\hline & Personalisation & Codification \\
\hline Reach & Whole organisation & Whole organisation \\
\hline Search Process & Contact an expert & $\begin{array}{l}\text { Search, review and use documents from } \\
\text { Knowledge Management Systems }\end{array}$ \\
\hline Transfer & Via person & Via document \\
\hline Memory & Present employees & Past and present employees \\
\hline Requirements & Expert directory & Creation and management of KMS \\
\hline Knowledge focus & Undocumented \& Documented & Documented \\
\hline Performance impact & Innovation \& Quality & Time saving \\
\hline
\end{tabular}

Which of the two approaches should be adopted by a company? On this issue, the literature provides interesting insights. We will especially focus on three points:

1. the relationship of the strategic, structural, and environmental conditions of a company with the KM approach that it adopts;

2. the prevalence (if any) of one of the two approaches (i.e. codification or personalization);

3. the changes of approach in the same company over time.

As regards the first point, it is generally agreed that there should be an alignment of the KM strategy adopted by a company - and, therefore, the choice between codification and personalization - with its business strategy, organizational characteristics, and also environmental conditions (Hansen et al., 1999; Koenig, 2001; McMahon et al., 2004; Scheepers, 2004; Greiner et al., 2007; Venkitachalam and Willmott, 2015; 2016). It must, however, be noticed that the various authors sometimes underline different factors as key elements to explain the adoption of a specific KM strategic approach (Table 2). Therefore, the overall picture is quite variegated. 
As regards the prevalence of one or the other KM approach - and especially, whether one of the two approaches should necessarily prevail in an organization, or if they can be adopted simultaneously - the opinions are not completely uniform. Codification and personalization were deemed to be opposite approaches and, at least initially, it was argued that companies should give a clear priority to one of them in their KM strategy. In a famous paper, Hansen et al. (1999) - hereafter HNT -propose that, although a totally exclusive adoption of either codification or personalization may be difficult and even unwise, a company should still prefer a KM strategy where one is strongly predominant and the other just supportive (specifically, they indicated a "desirable" 80-20 split). According to these authors, the choice of a clearly prevailing approach must be driven by the company's competitive strategy, and particularly by factors such as: the kind of product (standardised or customised), the level of innovativeness of their market offer (mature or innovative products), and the prevailing nature of knowledge employed by the staff to solve problems (explicit or tacit). In this view, a business strategy based on mature (and more standardized) products may benefit from a reuse model (i.e. a prevailing codification approach), while one based on innovative products may be favoured by a prevailing personalisation approach.

Table 2: Factors affecting the KM strategy mix identified by the literature

\begin{tabular}{|l|l|}
\hline Factor & Authors \\
\hline Business strategy & Koenig (2001); Hansen et al. (1999); Greiner et al. (2007); Ajith Kumar and Ganesh (2011) \\
\hline Competition & Venkitachalam and Willmott (2015; 2016) \\
\hline Organisational structure & Venkitachalam and Willmott (2016) \\
\hline Leadership & Venkitachalam and Willmott (2015) \\
\hline Organisational politics & Scheepers et al. (2004); Venkitachalam and Willmott (2015) \\
\hline Cultural context & Scheepers et al. (2004); Kumar and Ganesh (2011); Venkitachalam and Willmott (2015) \\
\hline Product characteristics & Hansen et al. (1999); McMahon et al. (2004); Venkitachalam and Willmott (2016) \\
\hline Business nature & Scheepers et al. (2004); Venkitachalam and Willmott (2016) \\
\hline Size & $\begin{array}{l}\text { Scheepers et al. (2004); Ajith Kumar and Ganesh (2011); Venkitachalam and Willmott } \\
\text { (2016); Powell and Ambrosini (2012) }\end{array}$ \\
\hline Geography & $\begin{array}{l}\text { Scheepers et al. (2004); Powell and Ambrosini (2012); Venkitachalam and Willmott } \\
\text { (2016) }\end{array}$ \\
\hline Technology (IT) & McMahon et al. (2004); Ng et al. (2012); Venkitachalam and Willmott (2015; 2016) \\
\hline Past experience & Powell and Ambrosini (2012) \\
\hline $\begin{array}{l}\text { Tacit vs. explicit } \\
\text { knowledge }\end{array}$ & Hansen et al. (1999) \\
\hline
\end{tabular}

Other studies clarified that a clear prevalence of personalization or codification approach in a company may be not always the best choice, and there can be different "shades" of KM approach even within the same organization. Koenig (2001), for instance, warns against falling for a "false dichotomy" and affirms that it may be dangerous to limit the combination of codification and personalization to a strict "80-20" proportion (as HNT substantially suggested). The outcomes of his analysis of the pharmaceutical industry show that a "correct" balance for the overall KM implementation may lie anywhere within the 80-20 to 20-80 range, and even in the same company, he argues that this proportion may change across different organizational functions or units. McMahon et al. (2004) also affirm that both approaches can be necessarily applied at the same time. Similarly, on the basis of an analysis of four companies operating in different industries, Scheepers et al. (2004) confirm that the "80-20 split" is not (necessarily) the best mix. Ng et al. (2012) are also in favour of a "hybrid/integrated strategy" where there is a substantial balance of the two approaches.

Greiner et al. (2007) agree with HNT that a prevailing personalization strategy can be more appropriate for organisations focusing on innovation, since they face high equivocality, while organisations that pursue efficiency may face less equivocality and, consequently, tend to adopt a prevailing codification strategy. However, in their view, codification and personalisation may and should be more mixed together, since simply relying on a strongly prevailing approach may be not always appropriate: an excessive emphasis on codification and reuse of knowledge may not allow to face market dynamics, while focusing on promoting interpersonal interactions does not necessarily lead to innovation. Indeed, the authors deem that if organisations rely too much on human factors, the new ideas may evaporate due to lack of actions and tools to harness them; while, if organisations emphasise formalized KM systems too much, creativity might be 
restrained by prearranged procedures and guidelines. Similarly, Venkitachalam and Willmott (2017) warn about the possible risks of an excessive personalization or codification: too much personalization may lead to "knowledge proliferation" while too much codification to "knowledge structuration" that can impede idea generation and radical innovations.

Ajith Kumar and Ganesh (2011) confirm that there may be a reinforcing relationship between the two KM strategies. Hence, the authors consider crucial to find a right balance of the two approaches because it can allow achieving the benefits of both reusing explicit knowledge and generating or disseminating the employees' tacit knowledge.

The analysis by Powell and Ambrosini (2012) of five real-life cases of management consulting companies shows that those companies can adopt what they define a "pluralistic approach" to KM (i.e. an approach that employs different KM tools and practices simultaneously) which can lead to personalization and codification without focusing predominantly on one of them. The organisations they studied perceived the pluralistic approach to KM as more effective than a single approach where personalization or codification strictly prevails. The authors argue that a "bespoke" approach (i.e. an approach where different systems are used in combination to manage different types of knowledge) can give better results since it allows to consider a firm's variegated cognitive needs.

As regards the third point - namely, if a company can change its KM approach over time - some studies confirm this possibility. Scheepers et al. (2004) underline that the combination of codification and personalization may not remain constant over time for the same company: organisations can find it useful (when not necessary) to modify their KM strategy time by time, to ensure an effective use of knowledge. They propose a model of "progressive strategic pathway" towards the effective use of knowledge, which is driven by factors as: the nature of the business, the political and cultural environment, the organisation size and location. For all these reasons, these authors affirm that future research should make use of longitudinal studies that closely track the temporal evolution of KM strategies in the same company and can therefore provide explanations of changes in the approach to KM over time.

As Venkitachalam and Willmott (2015; 2016) underline, organizations can have a different mix of personalization and codification depending on the interaction of the various internal and external environmental factors: since these can change over time, it is likely that the mix also changes.

Indeed, Powell and Ambrosini (2012) illustrate some cases of companies where the KM approach changed over time, and they underline that these changes were due to both modifications of contextual factors (such as firm size or geographic scope) and to the experience gained by the company from implementing a specific KM strategy. The KM approaches can, therefore, evolve dynamically: for example, companies can learn from their initial experience with formal KM practices and can later refine and redirect their approach. Moll (2019) explicitly considers the temporal evolution of KM programs in a large organization (NASA) from the 80s until today. The study shows how their journey started from highly relying on KM personalisation in the 1980s, to an increased emphasis on codification in the 1990s, to a more balanced approach starting from 2010. Moreover, the research shows that the different NASA centres are characterised by a different blending of the two strategies, which confirms that the KM strategy mix is dynamically affected by the specific context where it is implemented. All these studies offer some insights into how the KM strategy evolved in some companies. Generally speaking, further retrospective and longitudinal studies (Ajith Kumar and Ganesh, 2011) can provide a better understanding of how (and why) a company's KM approach can change over time.

To sum up, the literature review reveals that:

1. rather than a KM strategy based on just one (or on a clearly predominant) approach of personalization or codification, companies often adopt a combination of the two;

2. the adoption of a particular combination of the two approaches is influenced on the specific strategic, organizational, and environmental context of a company;

3. the KM strategy mix can change over time for the same company, as the context changes.

However, the literature, as we have shown, is quite fragmented and there is the need for fresh research that addresses all these three points contextually. Especially, it is useful to investigate how companies change their KM strategy over time, and what factors play a role in these changes. 


\section{Research goals and method}

Based on what was discussed in the previous section, the goal of this study is to investigate the long journey of a company in its KM strategy. Especially, we formulated two research questions, as follows:

RQ1: Can the mix of codification and personalisation KM strategies change over the years in the same company?

RQ2: If yes, how can these changes be explained in relation to specific organizational or environmental factors?"

In order to answer these questions, a case study methodology was adopted (Yin, 2018), that combined the use of a longitudinal study (based on frequent interviews with KM officers and direct analysis of KM programs of the company during almost a decade) and a retrospective study (based on documents and information derived from informed employees). Longitudinal research involves an extended time period to enable in-depth exploration and analysis of social phenomena, as these develop or change. It is mainly employed to search for explanations or clarifications by identifying patterns emerging over time, either within a single case or across cases in a comparative case study design (Mills et al., 2010). The investigated case concerns the KM initiatives undertaken during the last thirty years by the Advisory Service Line of a Global Consulting Company, whose name is disguised for reasons of confidentiality. The Company is a pioneer in the KM field, which facilitated the collection of information about their multi-year experience. It is a paradigmatic case: the company has also won the Global MAKE (Most Admired Knowledge Enterprise) award many times.

Since we intended to investigate the modification of the KM strategy mix and the related contextual changes that have occurred over approximately 30 years, it was impossible to perform a complete longitudinal study covering such a long period, so we integrated it with the retrospective study of the early KM experience of the company through available internal documents, public sources (such as: Book chapters, Academic journals and Conference papers, Case studies, public presentations, etc. - these were not included in the references for the above-mentioned confidentiality reasons but further details can be asked to the authors). Also, we made use of information provided by a senior KM manager who took an active part in the implementation and management of KM practices and tools adopted by the organisation during its whole KM journey.

To answer the research questions in a punctual way, the history was reconstructed by analysing the influencing factors identified by the literature and illustrated in Table 2, i.e.: competition, business strategy, product characteristics, managerial style, culture, KM perception, and enabling technologies. The KM strategy of the company was identified by describing the overall approach followed, the dominant KM processes, the KM technologies adopted, the nature of the KM sponsor and of the location mix, the prevailing kind of managed knowledge, the KM skills required.

\subsection{The Company}

The investigated case (in the following pages, we will simply refer to it as "the Company") is a global service company operating in more than 150 countries with almost 300,000 employees. It provides professional, consulting, and advisory services to business clients. As previously said, it is a pioneer in KM, and it has been integrating KM formal practices into its culture, processes, and infrastructure for more than twenty-five years. The Company has progressively developed its KM program from initially separated and locally based KM teams to a really "global" KM organization. The study focuses on the KM history of one of the company Business Units, the Advisory Service Line, which will be briefly summarised with the aim of describing how the KM strategy has changed over three decades, from mid-1990s to present.

\section{Case study}

\subsection{The 90s}

In the 90s, the overall market landscape and competition were changing fast, from local country-based services to more globalised products, services and skills. The emerging need for specialization led to more industrialized and efficient service delivery, designed for each specific sector. The managerial approach was very hierarchical, based on central decision-making, but with an ability to operate at the local level and in each specific area. The leadership style was quite traditional, with younger generations of professionals following 
the successful models of the past. There also was a strong influence of local cultural factors, which influenced behaviours and hindered transversal collaborations.

At that time, KM was considered a promising approach. In other (big) companies, KM programs were being developed as a way to support business strategies by means of a formalization of knowledge, for making it possible to share and reuse best practices across the organization. The available (and, at that time, emerging) technologies were centred on repositories and content management systems, whose goal was mostly to support an efficient distribution of data and databases. The first Intranet systems were being developed in those years, and they raised the attention especially of innovative companies, which were seeking for breakthrough technical solutions to get competitive advantage. Search engine technologies also started to improve gradually, and their efficiency became evident with the increase in the available digital content, not only in the internal databases but also externally.

The KM approach of the Company highly focused on reusing knowledge already available, firstly by identifying what was produced in the different locations, and then by codifying and storing these contents to make them easily and quickly available to a broader audience. Some "subject-driven" knowledge bases were implemented by collecting best practices in a central location and replicating it to other locations. However, there was an increasing awareness that a generic knowledge base may not be enough to sustain a business strategy of specialization, and that the existing search engines were not able to meet user needs. By the end of the decade, the first global taxonomies (i.e. a shared terminology used to tag contents) started to be created to both address the need for improved KM processes (searching/locating knowledge) and at the same time avoid fragmentation of intents (initiatives). In these years, the first "Community of Interest" networks were also established, where individuals physically met to agree on knowledge sharing and collaboration guiding principles and priority contents.

KM programs were organized by following what was, apparently, a double-faceted approach. Local KM teams and "service centres", delivering specific functions locally, were established by national offices following a standard approach centrally developed. In other words, the Company's approach to KM was centralized to ensure general coordination, while the knowledge content itself (and its exploitation) remained decentralised. Indeed, KM programs were sponsored typically by local consulting leaders, and knowledge managers usually sat at a local level, with limited hubs in strategic locations - near to influential leaders - like in the US and UK. Initially, prudent trials and extensions at a global level were promoted, to support global initiatives and to face general issues. Later, a central supporting service function was established and given an official status (1996), with the purpose to implement the KM infrastructure: a relatively centralised KM system build and managed form the top.

The KM approach regarded content collection, storage, and search on behalf of practitioners, in some cases with customized solutions. They were based on mature technologies (such as Lotus Notes and Domino, market leaders and de facto standards) adopted by the whole company to implement robust content management processes and enable appropriate Intranet user experience. Specific modalities of collecting, formatting, storing, and retrieving contents were also developed and applied, to ensure efficiency of KM re-using cycle, and the effective experience by users.

In conclusion, it can be argued that the overall KM strategy was substantially codification, to implement an abstract knowledge production process. The main goals were to improve and increase storage and reuse of key contents. In this framework, there was a clear emphasis on the diffusion of explicit knowledge. The most requested competencies for KM were those of librarians, humanistic disciplines, and communication-related degrees, essential to create the shared terminology that serves to facilitate the storage and retrieval of content.

\subsection{The '00s}

This decade showed a serious economic crisis due to the bursting of the dotcom bubble at the start, and the financial meltdown near the end and, therefore, increased competition in all industries including business services. In the consulting sector, there was a fierce selection of existing companies by market forces. The big players needed to become more efficient but were unable to prevent newcomers from entering the market, where they could take advantage of a smaller size and bigger agility to adapt to the fast-changing business conditions. Consulting branches of the "Big Four" companies were also sold, to overcome the "no- 
independence risk" connected to the conflict of interests that could raise when an audit company sells consulting services to the audited organization. However, these actions of spin-off led to a significant loss of intellectual capital. Furthermore, the lost competencies had to be developed again, because at the end of noncompete agreements, the spin-offs would be able to compete for the same clients and in the delivery of the same services. This competence-rebuilding process was achieved by hiring a team of experts whose tacit knowledge was used to introduce new service delivery methods and, in this way, transferred at a global level to be applied in the field. A common language definition was necessary, which resulted in long-lasting consultations at top management level and broad investments at global level, to improve efficiency and accelerate processes. Service globalization and specialization continued, with a process of dissemination of the experience made with pilot clients across other sectors, and mutual exchange of knowledge among them. Good examples about that are the application of best practices in Finance coming from the Utilities service sector, or the Supply chain best practices coming from the Manufacturing sectors.

Leadership and managerial style also changed. From a strongly vertical and functional hierarchy, it changed into a structure of multifunctional process-driven project teams. KM initiatives had a diverse mix of sponsorships, with some supporters but also sceptics. Generally speaking, in those years, KM initiatives lived a phase of general lack of trust, due to the failures of some programs in the previous decade and the poor results of the big investments in "hard" information technology. Indeed, the technological scenario was changing due to emergence of innovative Web 2.0 enabling technologies, which were progressively replacing the old information systems. "Social" applications made it possible to improve the engagement and the participation of final users, who became more involved in KM programs: they started functioning as active "producers and users" of knowledge contents rather than just passive users of the system. In those years, the Company also tested the first examples of smart working and virtual teaming.

In this overall scenario, the KM approach followed by the company still focused on reuse and sharing of knowledge: however, the focus was not only on explicit knowledge (as was in the previous decade, essentially), but also on tacit contents. Consequently, a twofold strategy was followed. On the one hand, to face the increasing need for a global approach to service delivery, KM programs were targeted to sustain the creation of a global language, aimed at accelerating and bringing efficiency to codified knowledge sharing. The Intranet system was renewed, with a focus on the creation and sharing of knowledge contents globally, although with a progressive consolidation of local practices at the same time. On the other hand, this strategy was complemented by personalization: the first online Communities of Practice were established to improve knowledge sharing by means of "social" interactions. On the whole, the KM approach resulted to be fragmented, with the major headquarters taking the lead and connecting to each other, while smaller branches still lacked a common strategic vision, and a structured plan of investments and resources. KM priorities were mainly directed to the definition of global taxonomies of knowledge contents, and on the creation of knowledge objects to be shared; at the same time, the establishment of the first online communities of practice was initiated. These communities gradually extended their geographical scope from single countries or regions to broader areas.

By the end of the decade, a new global KM platform was released. This new version strongly focused on user experience, with a combination of different content categories, and especially referring to methods and policies for "go-to-market" and service delivery, with a multiple view across all organizational domains. The system included the possibility to navigate through every content type referring to a specific sector, because a "sector tag" was added to classify each single document. Videoconferencing was also used extensively, to integrate leveraged explicit knowledge with stronger people-to-people tacit knowledge sharing.

The promoters of KM initiatives were no longer only local, with an involvement of regional and finally global executives. The global initiatives were typically led by the vertical dimensions of sectors where it was easier to establish a common language, while at the level of the single service delivery, approaches were still localized and fragmented. KM teams were organized at a regional level with a central shared service in some locations, providing a buffer of resources to address capacity peaks and back-end platform maintenance processes including e.g. document validation and publication.

So, while a codification strategy was still prevalent to sustain the continuous effort towards standardization, in parallel there was an emerging need for personalization, seen as incubator of new approaches and best practices. 
As mentioned before, there was more balance between explicit and tacit KM, with increased awareness that only the combination of the two kinds could result in a positive user experience and a more efficient reuse of existing intellectual capital. Summing up, while in the previous decade the prevalent focus was on content, in this decade there was a clear move towards community support and empowerment. The skills requested to knowledge managers started including more economic and managerial aspects, to improve process efficiency and metrics analysis to measure the impact of $\mathrm{KM}$. In addition, an effort to assess the effectiveness of communities of practice (for example, by means of questionnaires to members) was also implemented.

\subsection{The last decade}

This decade was characterized by a perception of more intense and faster market changes in the company's sector. Competition became more and more fierce. In this context, the business strategy focused on growth that could not be only internal but required acquisition of emerging and fast changing skills and competencies provided by new players and start-ups. These initiatives have implied embedding the knowledge assimilated from the acquired companies into proprietary assets. In addition, the first social platforms (based on technologies like Sharepoint or services like Yammer) were developed, where different players can collaborate and produce innovations in services and their delivery, to accelerate the "go-to-market" of new services and products. Online global communities were systematically launched, so that it became possible to mobilize know-how and experts in a fast and effective way, while simultaneously adopting and integrating their knowledge. Technological platforms were also redesigned to increase the use of self-service knowledge.

Managerial approaches changed consequently: more delegation and agility were allowed, organization becomes flatter, and leaders were recognized "on the field" rather than just through organizational charts. Human resources were characterized by the co-existence of different "generations". This claimed for a combination of traditional communication and management strategies with more innovative styles, and additional investments and resources were needed. Especially, the younger generations of employees were able to manipulate information more easily with technologies, while the older struggled to keep up with the changes and run the risk of being excluded by career progression if not well equipped on the technological side.

The technology-enabled KM processes lived a moment of renaissance, as they were recognized as being the foundational element of any socially enabled enterprise, the backbone of any new initiative. Especially, in this scenario, social platforms and networking systems emerged, disrupted the old behaviours and facilitated people-to-people interactions, empowering potentially anybody in the organization to raise questions and get an answer in few minutes if not seconds.

The main KM approach of the company shifted to channelling know-how and expertise where they are required as quick as possible, to leveraging and combining skilled people knowledge in order to keep up with a continuing evolving business. Online communities established in the late '90 were now revised and progressively evolved around the emerging social styles and social platforms. The same person could belong to different communities at the same time depending on roles, projects and interests. There was even a stronger pressure to improve the personal experience of each user, to the point that most of knowledge consumption now happens in a self-service mode. Knowledge services focused on cultural aspects, with the goal to promote a deep behavioural change of practitioners. Accordingly, content and community management got integrated. The results themselves of KM activities were evaluated based on the cultural changes they may induce, rather than on more "tangible" aspects. It was time for technology harmonization, where traditional tools got coupled with innovative social platforms. Knowledge was recognized as a key pillar of market strategy and was all managed at the global level, with a global sponsor and budget, and multi-location and multi-specialization shared services. There was a substantial balance and integration between codification and personalization: codification got progressively efficient by means of automation and was driven by data analytics, while personalization was used to promote and support the diffusion of a knowledge sharing-oriented culture. It was also the time for better balance and integration of explicit and tacit knowledge sharing, enabled by the abovementioned technologies. The KM skills that were increasingly considered to be essential included those related to computer science, engineering, analytics.

\section{Discussion}


Tables 3 and 4 provide a summary of the dynamic evolution of the KM approaches adopted by the case company, and of the enabling factors. Additional clarifications and details are also provided.

Table 3: Summary of findings: strategic and organizational factors

\begin{tabular}{|l|c|c|c|}
\hline Factor & 1990s & 2000s & 2010s \\
\hline Competition & Globalisation and dynamism & Crisis and fierce competition & Continuous evolution \\
\hline $\begin{array}{l}\text { Business } \\
\text { strategy }\end{array}$ & Specialization & Business restructuring & Growth \\
\hline Product & $\begin{array}{c}\text { Local specialized services for } \\
\text { specific sector }\end{array}$ & $\begin{array}{c}\text { More and more specialized and } \\
\text { global }\end{array}$ & Global platforms \\
\hline Managerial style & Hierarchical & Process-based & Participatory \\
\hline Culture & $\begin{array}{r}\text { Homogeneous by geographical } \\
\text { area }\end{array}$ & Heterogeneous & Heterogeneous \\
\hline KM perception & Trendy & Scepticism & Renaissance \\
\hline $\begin{array}{l}\text { Enabling } \\
\text { technologies }\end{array}$ & Content repositories & $\begin{array}{c}\text { User generated content } \\
\text { repositories }\end{array}$ & Social networks \\
\hline
\end{tabular}

Table 4: Summary of findings: KM approaches

\begin{tabular}{|c|c|c|c|}
\hline KM & $1990 s$ & $2000 s$ & 2010s \\
\hline Overall approach & Storage and re-use & From re-use to sharing & $\begin{array}{l}\text { Sharing/creating new } \\
\text { knowledge }\end{array}$ \\
\hline KM processes & Content management & $\begin{array}{l}\text { Content management and } \\
\text { sharing of tacit knowledge }\end{array}$ & Dynamic knowledge sharing \\
\hline KM technology & Databases and search engines & Repositories and networking & Social media platforms \\
\hline KM sponsor & $\begin{array}{c}\text { Local (single initiatives) and } \\
\text { global (general coordination } \\
\text { and standardization) }\end{array}$ & $\begin{array}{c}\text { From local, to regional, to } \\
\text { multi-regional, to global }\end{array}$ & Global \\
\hline KM location mix & $\begin{array}{l}\text { Local (single initiatives) and } \\
\text { global (coordination) }\end{array}$ & $\begin{array}{c}\text { (Fragmented) mix of local and } \\
\text { global }\end{array}$ & Global \\
\hline $\begin{array}{l}\text { Codification Vs. } \\
\text { personalisation }\end{array}$ & Codification prevails & $\begin{array}{l}\text { Codification still prevails, but } \\
\text { personalization goals increase }\end{array}$ & Substantial balance \\
\hline Explicit Vs. tacit & Prevalently explicit & $\begin{array}{c}\text { More balanced between tacit } \\
\text { and explicit }\end{array}$ & $\begin{array}{c}\text { Substantial balance between } \\
\text { tacit and explicit }\end{array}$ \\
\hline $\mathrm{KM}$ focus & Content & Content + Community & $\begin{array}{c}\text { Content }+ \text { Community + Culture } \\
\text { of sharing }\end{array}$ \\
\hline KM skills mix & $\begin{array}{c}\text { Librarians, graduates in Human } \\
\text { Sciences (communication and } \\
\text { language skills) }\end{array}$ & Economists & $\begin{array}{c}\text { Engineers, computer scientists, } \\
\text { data analysts }\end{array}$ \\
\hline
\end{tabular}

What emerged from the investigated case provides a positive answer to both our research questions. The study also confirms that companies may tend to adopt a balanced strategic mix, i.e. they keep searching for the most appropriate combination and integration of codification and personalisation; this is contingent on a set of internal and external factors and can vary over time.

Concerning the first research question (i.e. if it is confirmed that the mix of codification and personalisation knowledge strategies can change over the years for the same company), the evolution of the KM strategy of the investigated company shows that this is exactly the case. In particular, the company has followed a path moving from an initially codification-oriented approach to a more balanced one. The company has moved from a locally fragmented KM to a globally managed one. It is interesting to note that, at some points in time, one or the other approach tends to prevail due to development of specific initiatives (e.g. the creation of taxonomies or the restructuring of communities). However, if we consider the entire period, there isn't a single and unchanging KM strategy, because the combination of personalization and codification has changed over time. Hence a first lesson from the study is that the evolution of a company's KM strategy follows a path denoted by a dynamic balance between codification and personalisation. 
As far as the second question is regarded (i.e. which factors can influence the changes in the KM strategic mix), the investigated case offers interesting insights. More specifically, it suggests that various factors have had different importance and played different roles. Those strictly related to the competitive environment, as the nature of competitors and markets, or the implemented business and product strategies, seem to have strongly affected the KM strategy mix, which confirms the results of previous studies (Hansen et al., 1999; Greiner et al., 2007): the choice is primarily driven by the company's business strategy. Other factors - like managerial style and leadership, culture and KM perception (Scheepers et al., 2000); Kumar and Ganesh, 2011; Venkitachalam and Willmott, 2015) seem to have acted as specific constraints or enablers that may, however, influence the way KM strategy is carried out. For instance, in the investigated case, in the '90s a not uniform propensity in the company towards innovative behaviours determined a territorially fragmented adoption of KM tools and practices, which confirms the importance of geographical localization in shaping the KM approach (Scheepers et al., 2004; Powell and Ambrosini, 2012; Venkitachalam and Willmott, 2016). Conversely, a more participative leadership style has progressively favoured the use of Communities of Practices.

Technology deserves a special mention since it seems to act as both a constraint and an opportunity, which is in line with the findings of the previous studies (McMahon et al., 2004; Ng et al., 2012; Venkitachalam and Willmott, 2015, 2016). While the first KM ICT-based systems of the '90s were well suited for a codification strategy, the new Web 2.0 tools, and specifically the Enterprise Social Media platforms introduced by the company, appear to be more "neutral" and to favour a mixed KM approach, for they can be used to manage both tacit and explicit knowledge. This last point, together with the findings of the recent literature (e.g. Venkitachalam and Willmott, 2017; Asher and Popper, 2019) raises the question whether the distinction between the two approaches and the elements on which they are based is always so sharp to be clearly used in practical terms, or is just a more generic reference. This point clearly deserves further analysis.

\section{Conclusion}

The study contributes to improve our understanding about the nature of the KM strategy mix and specifically its evolution and affecting factors.

As regards the academic contribution, it confirms the findings of previous literature about the dynamics of KM strategy mix and about the factors that influence such dynamics. Furthermore, it allows to distinguish the different factors on the basis of the role that they play in shaping this mix. Lastly, it raises the doubt that the distinction between codification and personalisation still has a practical sense.

Concerning the managerial contribution, the study re-affirms the importance of a balanced, but not static, KM approach, and offers food for thought to managers regarding the need to continuously align the KM strategy to internal and external environmental changes, and especially to the business strategy adopted to face such changes. In this regard, the study suggests that companies should learn to audit the changes of their business context by using a knowledge-based reading, i.e. by understanding the impact of such changes on the knowledge assets needed to preserve their competitiveness.

Clearly the study is not without limitations. Particularly, only one case (even if paradigmatic since the investigated company is one of the most admired in the KM field) may not be enough to generalise findings. Furthermore, it focused on a limited set of factors. This paves the way for future research aimed at deepening our understanding on the topic.

\section{References}

Ajith Kumar, J. and Ganesh, L.S., 2011. Balancing knowledge strategy: codification and personalization during product development. Journal of Knowledge Management, 15(1), pp 118-135.

Asher, D. and Popper, M., 2019. Tacit knowledge as a multilayer phenomenon: the "onion" model. The Learning Organization, 26(39), pp. 264-275.

Bolisani, E. and Bratianu, C., 2017. Knowledge strategy planning: an integrated approach to manage uncertainty, turbulence, and dynamics. Journal of Knowledge Management, 21(2), pp 233-253.

Bolisani, E. and Bratianu, C., 2018. Knowledge strategies. In: E. Bolisani and C. Bratianu (eds.) Emergent Knowledge Strategies. Strategic Thinking in Knowledge Management, Cham: Springer, pp 97-116

Coakes, E., Amar, A.D. and Granados, M.L, 2010. Knowledge management strategy, and technology: a global snapshot. Journal of Enterprise Information Management, 23(3), pp 282-304. 
Greiner, M.E., Böhmann, T. and Krcmar, H., 2007. A strategy for knowledge management. Journal of Knowledge Management, 11(6), pp 3-15.

Hansen, M.T., Nohria, N. and Tierney, T., 1999. What's your strategy for managing knowledge. Harvard Business Review, 77(2), pp 106-116.

Hislop, D., Bosua, R. and Helms, R., 2018. Knowledge management in organizations: a critical introduction, Oxford, UK: Oxford University Press.

Holsapple, C.W. and Jones, K., 2006. Knowledge management strategy formation. In: D. G. Schwartz (ed.), Encyclopedia of Knowledge Management, Hershey, PA: Idea Group, pp 419-428.

Koenig, M., 2001 Don't fall for that false dichotomy! Codification vs. personalization. KM World Magazine, 10(8). [online] Available at: https://www.kmworld.com/Articles/Editorial/Feature/Don\%E2\%80\%99t-fall-for-that-false-dichotomy!Codification-vs.-personalization--9272.aspx [Accessed 29 September 2020]

McMahon, C., Lowe, A. and Culley, S., 2004. Knowledge management in engineering design: personalization and codification. Journal of Engineering Design, 15(4), pp 307-325.

Mills, A.J., Eurepos, G. and Wiebe, E. (eds.), 2010. Encyclopedia of Case Study Research, Thousand Oaks, CA: Sage Publication.

Moll, J.K.Q., 2019. Personalization and codification at NASA: a case of an evolving knowledge management strategy. Master Thesis, Stellenbosch University, Stellenbosch, South Africa.

Ng, A.H.H., Yip, M.W., binti Din, S. and Bakar, N.A., 2012. Integrated knowledge management strategy: a preliminary literature review, Procedia-Social and Behavioral Sciences, 57, pp 209-214.

Nonaka, I. and Takeuchi, H., 1995. The knowledge-creating company. Oxford: Oxford University Press,

Polanyi M., 1966. Tacit Dimension, New York: Doubleday \& Co.

Powell, T.H. and Ambrosini, V., 2012. A pluralistic approach to knowledge management practices: evidence from consultancy companies. Long Range Planning, 45(2-3), pp 209-226.

Scheepers, R., Venkitachalam, K. and Gibbs, M.R., 2004. Knowledge strategy in organizations: refining the model of Hansen, Nohria and Tierney. The Journal of Strategic Information Systems, 13(3), pp 201-222.

Shannak, R.O., Ra'ed, M. and Ali, M., 2012. Knowledge management strategy building: literature review", European Scientific Journal, 8(15), pp 143-168.

Venkitachalam, K. and Willmott, H., 2015. Factors shaping organizational dynamics in strategic knowledge management. Knowledge Management Research \& Practice, 13(3), pp 344-359.

Venkitachalam, K. and Willmott, H., 2016. Determining strategic shifts between codification and personalization in operational environments. Journal of Strategy and Management, 9(1), pp 2-14.

Venkitachalam, K. and Willmott, H., 2017. Strategic knowledge management-Insights and pitfalls. International Journal of Information Management, 37(4), pp 313-316.

Yin, R.K., 2018. Case Study Research. Design and Methods, Thousand Oaks, CA: SAGE Publications, 6th ed. 